\title{
Modern Evaluation of Abdominal Trauma
}

Errington C. Thompson, $\mathrm{MD}^{1}$, Chadwick J. Knight, $\mathrm{MD}^{1}$

\section{Author Affiliations:}

1. Joan C Edwards School of Medicine, Marshall University, Huntington, West Virginia

The authors have no financial disclosures to declare and no conflicts of interest to report.

Corresponding Author:

Errington C. Thompson, MD, FACS, FCCM

Department of Surgery

Marshall University Joan C. Edwards School of Medicine

Huntington, West Virginia

Email: errington@erringtonthompson.com 


\section{Abstract}

Abdominal trauma can be mysterious to some physicians. If patients are evaluated for being stable or unstable, then abdominal trauma can be easily managed. Using a combination of physical examination, ultrasound and CT scans, patients can be quickly and efficiently evaluated.

\section{Keywords}

Trauma, abdominal trauma, ultrasound, wounds and injuries, blunt trauma, penetrating trauma

\section{Introduction}

The evaluation of the patient who presents with abdominal trauma has always presented a diagnostic and therapeutic challenge. In today's environment, the surgeon must weigh the risks and the benefits of performing versus not performing a variety of diagnostic and therapeutic procedures within the framework of what is safest for the patient. While exploratory laparotomy must be considered the gold standard for the evaluation and treatment of these patients and must be factored into their evaluation, clearly not every patient requires such a procedure. Abdominal exploration, while decreasing the risk of missed injury to near zero in the hands of an experienced surgeon, has been shown to have a complication rate from $15 \%-50 \%$ with an average length of hospital stay being a little over five days. ${ }^{1}$ Once considered a benign procedure, laparotomy must be reserved for those patients who will derive clear benefit. Noninvasive methods of evaluating the abdomen such as computed tomography and abdominal sonography have the advantage of delivering critical information at greater levels of comfort for the patient. The disadvantage of these non-invasive methods is the possibility of missing a critical intraabdominal injury and subsequent increased morbidity, mortality and cost. Diagnostic peritoneal lavage, which is being employed less frequently, lies in the mid-point of the spectrum of diagnostic modalities in terms of cost, invasiveness, sensitivity and specificity. In the last decade laparoscopy is finding its place in the diagnostic and therapeutic armamentarium of the trauma surgeon.

Our approach to the evaluation of the traumatized abdomen, while not unique, incorporates interventions that may not be universally available. In developing such an approach, it is important for the surgeon to be aware of the capabilities of his or her own institution. The following algorithm works when you have the readily available services of competent trauma and critical care surgeons, radiologists and continuously available operating suites staffed by experienced and capable personnel.

\section{Penetrating Abdominal Wounds-Stable}

Patients who present with penetrating anterior abdominal stab wounds are first confirmed to be hemodynamically stable rather than hemodynamically normal (multiple readings of heart rate under 100 and systolic blood pressure appropriate for age qualify as hemodynamically stable). (Figure 1) Patients in this category who are determined to have obvious peritoneal penetration are taken directly to the operating room for abdominal exploration. In patients without obvious peritoneal penetration, our preferred approach is emergency department wound exploration under local anesthesia with good lighting and exposure. This requires extension of the wound, adequate operative assistance and, occasionally, intravenous sedation. If peritoneal penetration 
cannot be determined, further exploration in the operating suite is then performed. If peritoneal penetration is confirmed, the patient should then undergo further workup - computed tomograms of the abdomen and pelvis or possible abdominal exploration. ${ }^{2}$ If peritoneal penetration can be definitively ruled out, the wound may be irrigated and closed and the patient discharged. It is possible to serially evaluate patients with equivocal signs of peritoneal penetration, but this requires a significant amount of time. ${ }^{3}$ Most physicians outside of institutions associated with a surgical residency program do not have the luxury of examining patients every two to four hours. A more aggressive alternative would be diagnostic laparoscopy in the operating room. This would eliminate the need for serial exams and would be less invasive than a formal laparotomy. This is a reasonable approach for patients with questionable peritoneal penetration. If an injury is encountered during a laparoscopic exploration it can be repaired either through the scope or by opening the patient. The goal must be to perform the safest operation for the patient. In our experience and environment prompt operative exploration is the best course of treatment for these patients.

There is mention in the literature of utilizing ultrasound for the evaluation of abdominal stab wound tracts. ${ }^{4}$ This can be very difficult and operator dependent. Unless the institution has a particular expertise and interest in utilizing sonography in this manner, we do not recommend nor use this technique.

Laparoscopy is an excellent tool to evaluate peritoneal perforation in cases where it is difficult to otherwise ascertain. In select patients, body habitus can make it difficult to determine if the tract of the penetrating injury parallels the peritoneal cavity or actually enters the abdomen. Instead of trying to open the entire length of the wound, laparoscopy can be employed to definitively determine the existence of peritoneal penetration.

We currently operate upon all patients who present with abdominal gunshot wounds. We have found laparoscopy very helpful in patients with presumed tangential abdominal gunshot wounds. With the laparoscope, the anterior and lateral abdominal walls can be completely inspected and if there is any evidence of peritoneal penetration, the abdomen can be opened and explored. After a negative laparoscopic evaluation, the patient can be started on a regular diet and discharged home within a few hours of the operation. There are several drawbacks to laparoscopy - expense, the right OR personnel, setup time, etc. ${ }^{5}$

More than a decade ago there was considerable interest in using the smaller 3-mm laparoscope in the emergency department. Stable patients with presumed tangential injuries underwent laparoscopy with intravenous sedation and narcotics. The scope was introduced under local anesthesia. Because of its analgesic properties nitrous oxide was used for abdominal insufflation. Unfortunately, the field of view for this scope was relatively small for adequate evaluation of the entire abdominal wall. There were also many logistical difficulties encountered in performing this highly advanced procedure in the emergency department, such as training ER personnel to perform an OR procedure. Finally, due to the cost of the scope, equipment and sterilization materials needed to be kept on standby for this relatively small subset of patients, the costeffectiveness of this approach was not realized and utilization of the 3-mm laparoscope in the emergency department for penetrating trauma has been largely abandoned. 


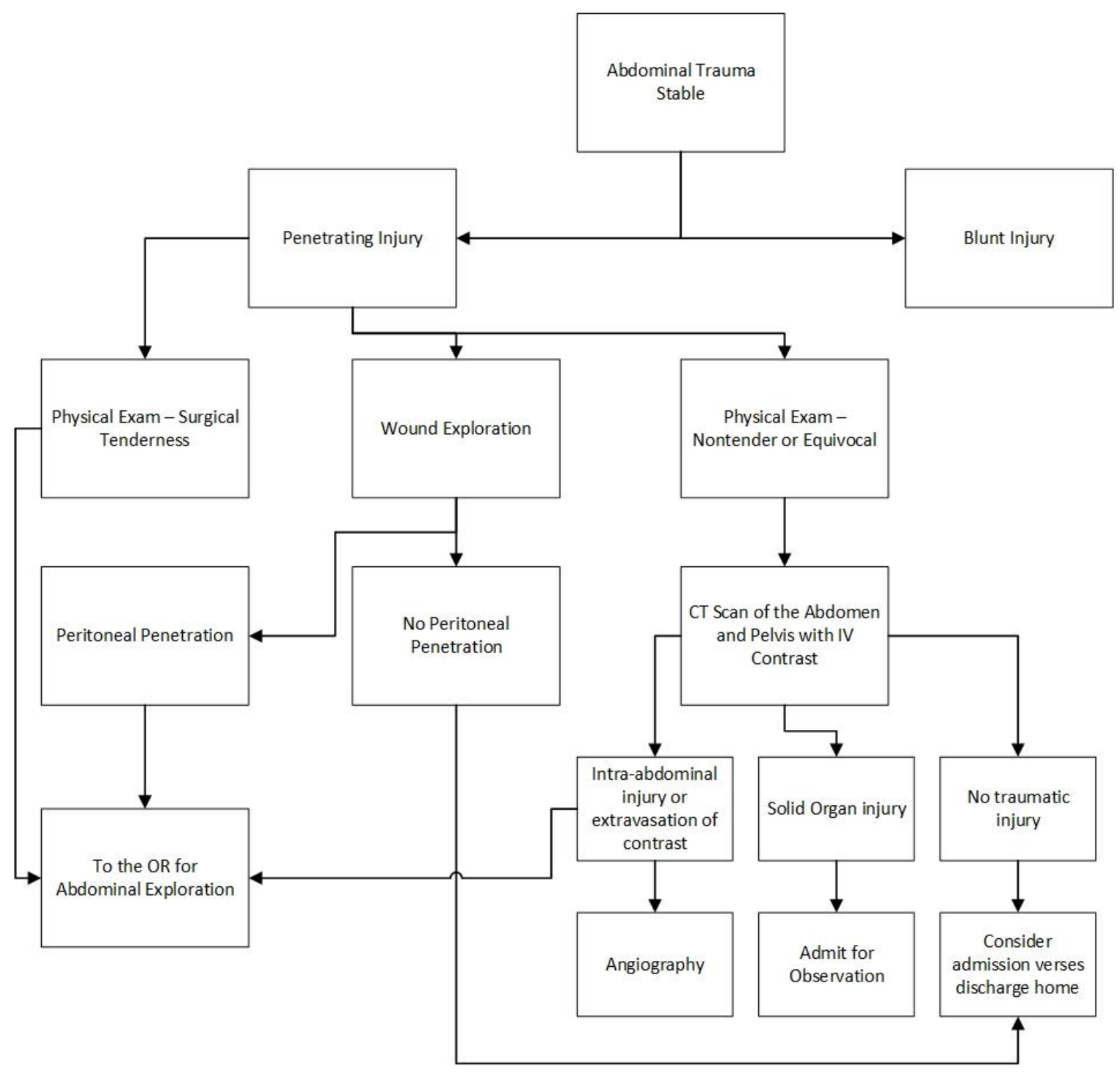

Figure 1: Penetrating Abdominal Trauma - Stable

\section{Penetrating Abdominal Wounds - Unstable}

Patients presenting to the emergency department who have suffered penetrating abdominal injury and manifest signs and symptoms of hemodynamic shock are taken expeditiously to the operating room for emergency laparotomy. (Figure 2) Advanced Trauma Life Support (ATLS) ${ }^{6}$ protocol should be followed in the emergency department. Two large bore IVs are started, blood is drawn for pertinent labs, to include a type and crossmatch. Some centers are utilizing O positive blood on patients who have not had prior blood transfusions or women of childbearing age. Patients who are Rh negative should not have an antibody to Rh positive cells unless they were previously transfused. Women of childbearing age is a special case in which an Rh negative 
mother can develop Rh positive antibodies if the maternal blood is exposed to the Rh positive baby. We continue to use $\mathrm{O}$ negative blood whenever available.

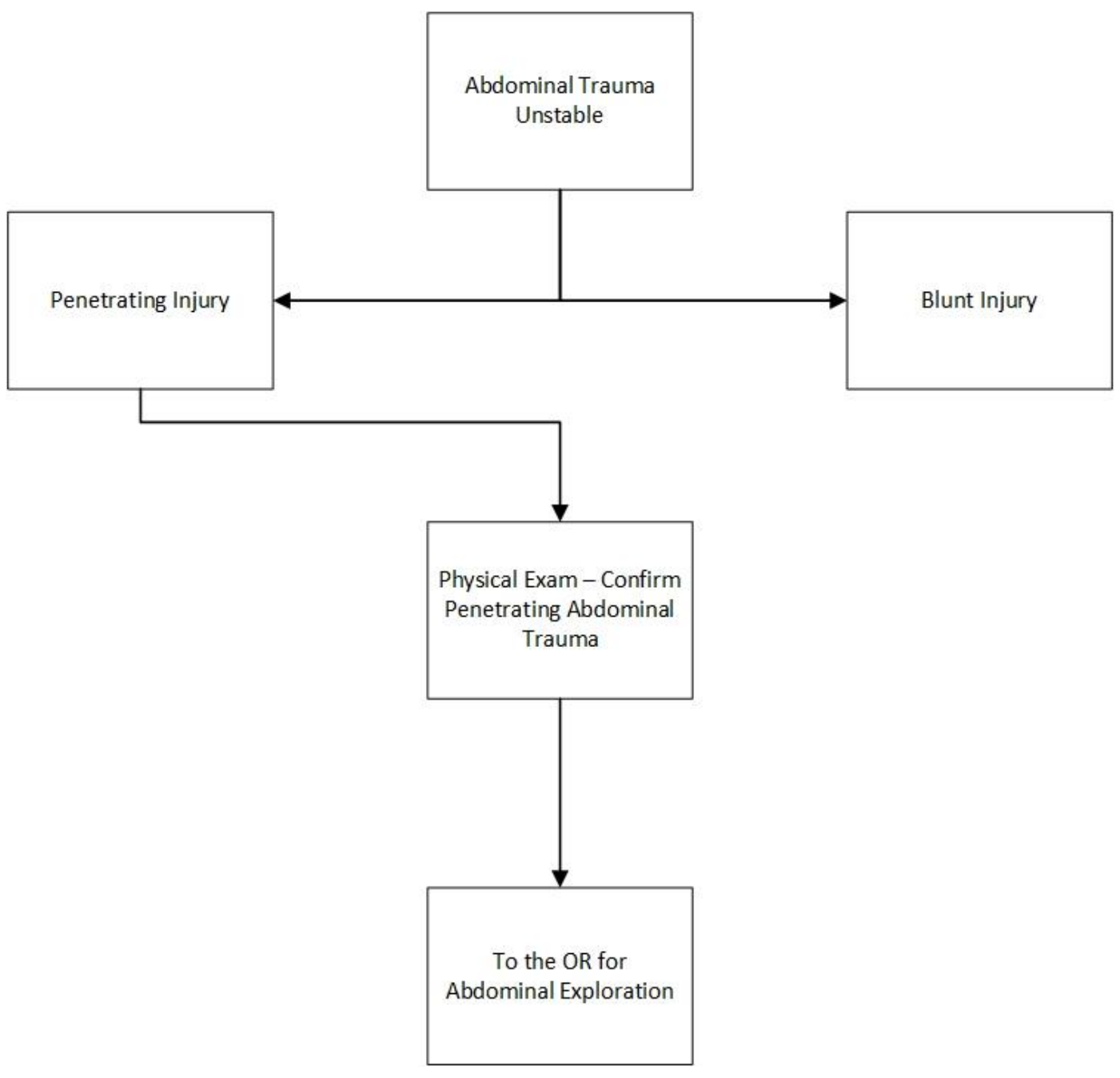

Figure 2 - Penetrating Abdominal Trauma - Unstable

We have not adopted delayed fluid resuscitation for penetrating torso trauma as advocated by Mattox, et al. ${ }^{7}$ This study found a significant decrease in mortality in the group of patients who had fluid resuscitation delayed until arrival in the operating room. All of the patients in the study had relatively short transport times from the field in Houston to the emergency department. In West Virginia, we do not have the luxury of short transport times. Over half of our patients come from surrounding areas. Transport times can vary from 10 minutes to several hours. This study also included only penetrating trauma, whereas the vast majority of our patients are blunt trauma victims. It is unclear whether Mattox's data can be translated to our patient population. Although 
we have not formally adopted delaying fluid resuscitation, we do believe in the overall concept that Mattox is advocating. Giving excessive fluids to patients whose bleeding is not controlled can elevate blood pressure and disrupt clots and dilute clotting factors which can cause further bleeding. Ideally, we would like to get patients to the operating room as quickly as possible and give patients only as much fluid as necessary to maintain adequate perfusion until the surgeon is able to stop the bleeding.

Quickly getting the patient to the operating room is of paramount importance. If the length of time that the patient is unstable can be shortened, morbidity and mortality can be decreased.

\section{Blunt Abdominal Trauma-Stable}

The stable patient with blunt abdominal trauma first undergoes physical examination. (Figure 3) In the patient with isolated abdominal trauma, physical examination is very reliable. If the patient has an altered mental status, a history of loss of consciousness or distracting injuries, then the physical exam can be less trustworthy. In these trauma patients, further workup is warranted. Ultrasound has been widely adopted by trauma surgeons as a tool to look into the abdomen for evidence of significant organ injury. The ultrasound is used not to specifically investigate organ architecture, but instead to look for blood. Blood acts as a surrogate for significant organ injury. Some authors have suggested that ultrasound be used as the initial screening modality in blunt trauma patients. ${ }^{8,9}$ One study has suggested that with as little as 10 ultrasound examinations, trauma surgeons have been able to accurately detect the presence or absence of fluid in the abdomen. ${ }^{10}$ Another study, specifically designed to look at how much fluid or how little fluid trauma surgeons can actually detect, found that the average surgeon required almost $600 \mathrm{~mL}$ of blood before fluid was detected. ${ }^{11}$ Even more concerning, fewer than $10 \%$ of the surgeons were able to detect less than $400 \mathrm{~mL}$ of blood. On the other hand, one of the best things about ultrasound is that it can be repeated quickly and easily anywhere in the hospital. Therefore, after an initial negative ultrasound if the patient's condition changes a repeat ultrasound can be done immediately.

Newer generation CT scanners can perform an examination of the abdomen and pelvis in 5 - 10 minutes. It is imperative that the trauma surgeon personally reviews the CTs as soon as they are formatted. Injuries to the liver, spleen, kidneys and bony pelvis are common. While most solid organ injuries can be managed non-operatively, active extravasation of contrast dye requires

prompt attention from the trauma surgeon. ${ }^{12}$ The trauma surgeon must decide whether the patient would be best served with some type of interventional radiology procedure or an open laparotomy in order to control the hemorrhage. Multiple factors must go into the surgeon's decision, including the availability of radiology, the availability of the operating room, the patient's hemodynamic status and the patient's other injuries. Extravasation requires some type of immediate intervention. 


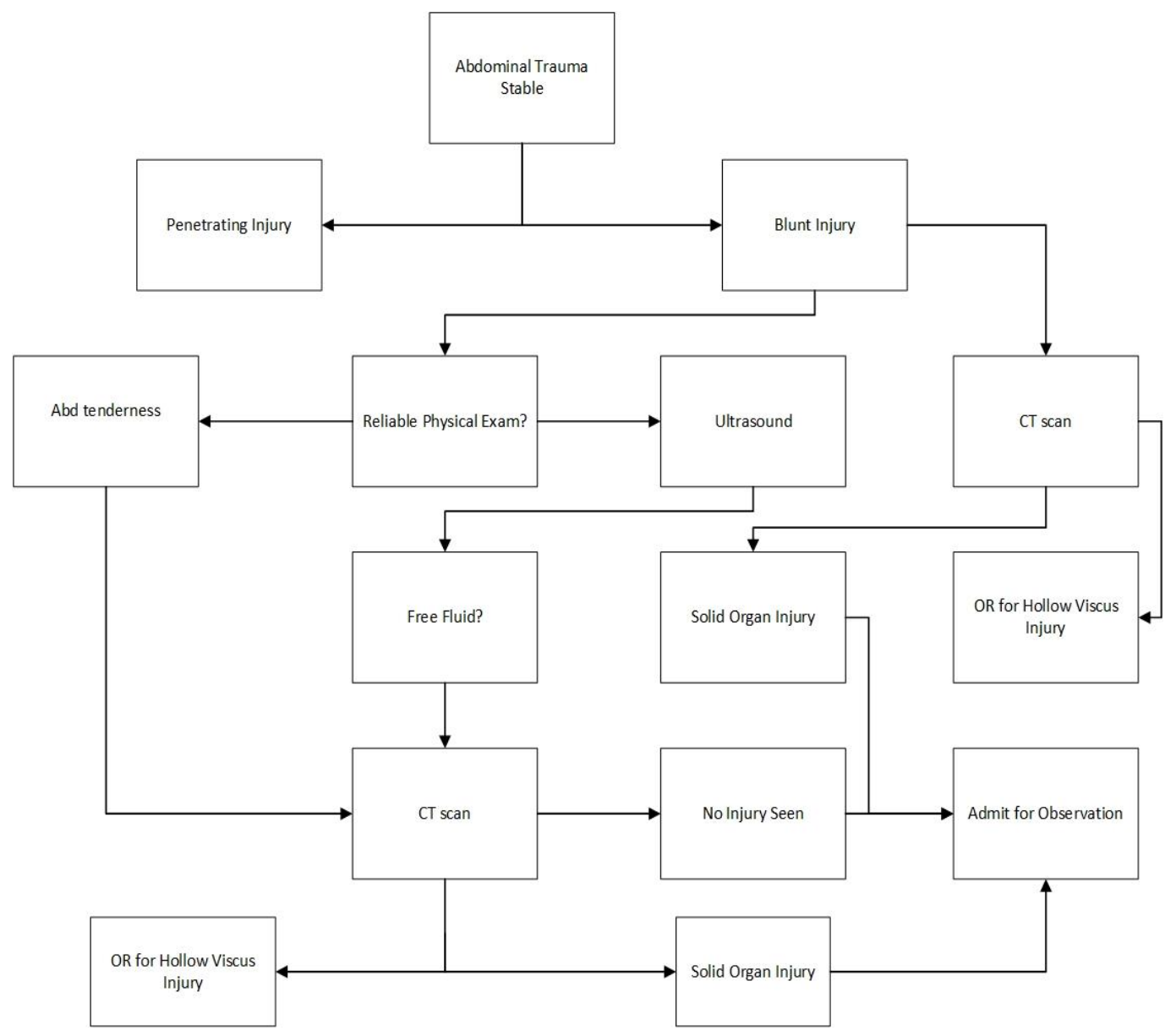

Figure 3 - Blunt Abdominal Trauma - Stable

\section{Blunt Abdominal Trauma-Unstable}

The majority of these patients are victims of motor vehicle crashes or motorcycle crashes and present with evidence of multisystem trauma. (Figure 4) Usually hypotension is caused by blood loss into the abdomen, chest, large bone fractures (pelvis, femur, open tibia/fibula) or from exsanguinating hemorrhage from an external wounds.

There are only three diagnostic modalities that we consider safe and proven effective in evaluating blunt abdominal trauma in the unstable patient - ultrasound, diagnostic peritoneal lavage (DPL) and exploratory laparotomy. Although ultrasound has been used in Europe for years to evaluate patients with abdominal trauma, only over the last 15 years have US surgeons embraced the ultrasound. Ultrasound is being used so frequently in these types of unstable patients that it should be considered a part of the physical exam. 


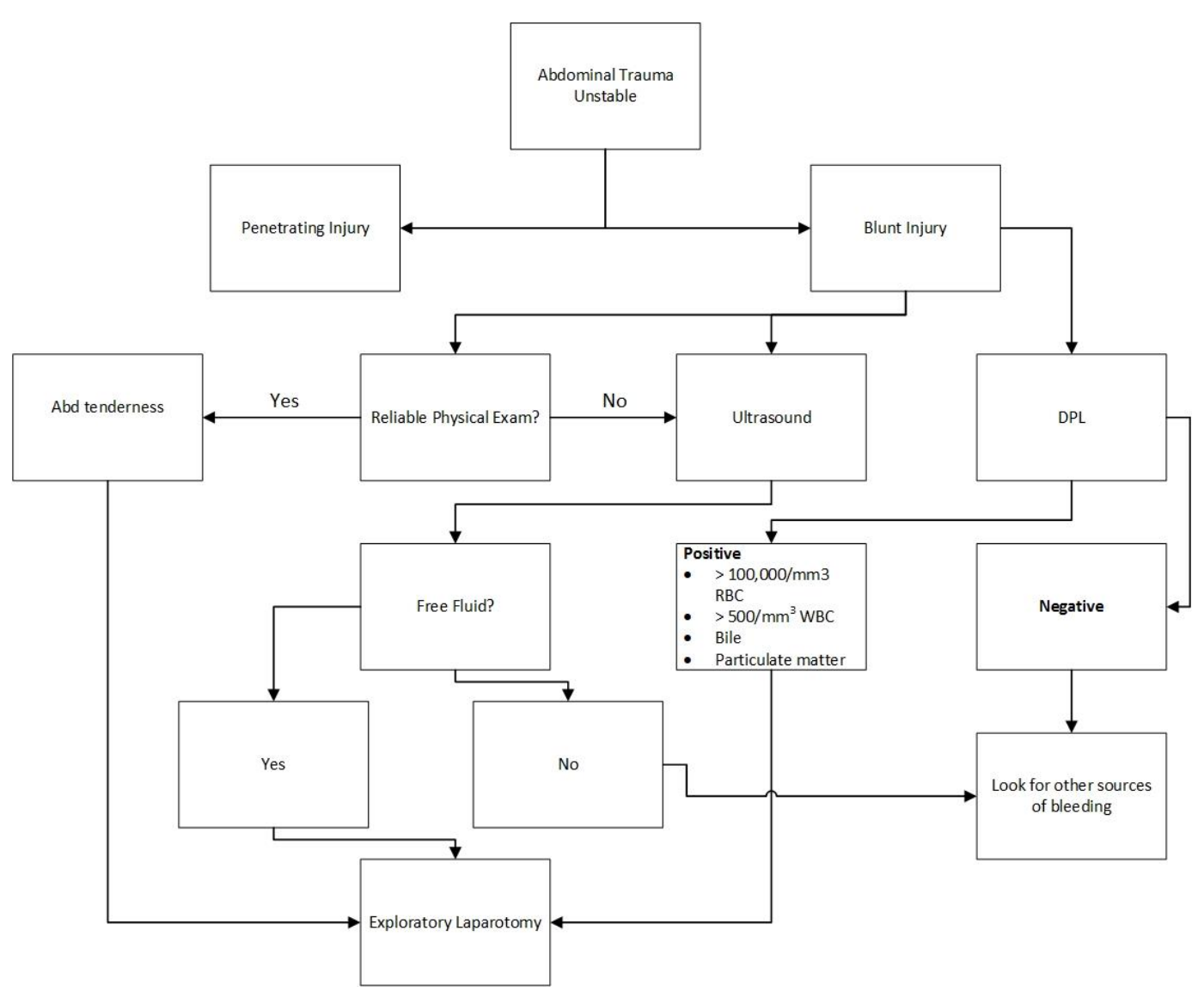

Figure 4 - Blunt Abdominal Trauma - Unstable

DPL is an excellent but invasive diagnostic tool. In the search for the cause of hypotension, a grossly positive result is helpful. Drawing back 5 - $10 \mathrm{cc}$ of frank blood mandates immediate surgery. Microscopic evidence of abdominal trauma is important but should not necessitate a patient's remaining unstable. The superiority of percutaneous versus open peritoneal lavage has been argued in the past. There is no study that we are aware of which demonstrates the dominance of one technique over the other. They both seem to be roughly equivalent. The open technique requires two people, excellent exposure and lighting. The closed technique is quicker and requires only one person to perform. The risk of iatrogenic bowel injury is greater with the percutaneous technique but is still less than $2 \%$ in skilled hands. One of the greatest dilemmas with the closed technique is what to do when less than 200-400 $\mathrm{cc}$ of an infused liter of saline is returned. A subsequent CT scan of the abdomen would reveal fluid that complicates the interpretation of this study enormously. Fluid is sometimes the only abnormal finding associated with small bowel injuries. Grading solid visceral injuries is also more difficult. This dilemma remains unresolved. ${ }^{13}$ DPL has really been used less and less by the modern trauma surgeon. Ultrasound is readily available in almost every ER in the US. DPL offers very few advantages over Focused Assessment with Sonography for Trauma (FAST).

Laparotomy, as mentioned earlier, is still an excellent way to evaluate the abdomen. In areas where severe abdominal trauma is rarely seen, ER ultrasound and DPL equipment may not be 
available. A rapid abdominal exploration may be the best course of action prior to obtaining further testing.

It should be mentioned that unstable patients do not go to the CT scanner for evaluation. Unstable patients must be quickly evaluated and converted from unstable to stable. This may require simply as little as a fluid bolus or as much as a trip to the operating room. Once the patient is stable a CT scan can be obtained to look for other associated injuries.

\section{Summary}

Evaluation of patients with abdominal trauma remains a challenging undertaking. These guidelines do not defer significantly from the Eastern Association for the Surgery of Trauma guidelines which were written in 2002 (blunt) ${ }^{14}$ and 2010 (penetrating) ${ }^{2}$. In this manuscript we have tried to emphasize ultrasound as noninvasive tool which can help the trauma surgeon with therapeutic decisions. We have included diagnostic peritoneal lavage for completeness. For the most part, the vast majority of trauma surgeons are not performing DPL's. There is no one right way to evaluate a patient with abdominal trauma. The approach needs to incorporate the status of the patient and the available resources within the institution. 


\section{References}

1. Sosa JL, Baker M, Puente I, Sims D, Sleeman D, Ginzburg E, Martin L: Negative laparotomy in abdominal gunshot wounds: potential impact of laparoscopy. J Trauma Acute Care Surg. 1995;38(2):194-7.

2. Como JJ, Bokhari F, Chiu WC, Duane TM, Holevar MR, Tandoh MA, Ivatury RR, Scalea TM, Practice Management Guidelines for Selective Nonoperative Management of Penetrating Abdominal Trauma. J Trauma Acute Care Surg. 2010;68:721-733.

3. Demetriades D, Velmahos G, Cornwell EE III, Berne TV, Cober S, Bhasin PS, Belzberg H, Asensio J. Selective nonoperative management of gunshot wounds of the anterior abdomen. Arch Surg 1997;132:178183.

4. Fry WR, Smith RS, Schneider JJ, Organ CH, Jr.: Ultrasonographic Examination of Wound Tracts. Arch Surg 1995; 130(6):605-7.

5. Block EFJ, Singh I, Thompson EC. Utility and cost-saving of diagnostic laparoscopy in low-probability gunshot wounds to the abdomen. Journal of the Louisiana State Medical Society. 1998;150: 232-234.

6. Initial Assessment and Management. Advanced Trauma Life Support for Doctors. $8^{\text {th }}$ Edition. Chicago: 2008. 1-18.

7. Bickell WH, Wall MJ, Pepe PE, Martin RR, Ginger VF, Allen MK, Mattox KL. Immediate versus delayed fluid resuscitation for hypotensive patients with penetrating torso injuries. N Engl J Med. 1994;331(17):1105-9.

8. Rozycki, G. S., Ochsner, M. G., Feliciano, D. V., Thomas, B., Boulanger, B. R., Davis, F. E., et al. Early detection of hemoperitoneum by ultrasound examination of the right upper quadrant: a multicenter study. Journal of Trauma and Acute Care Surgery. 1998; 45(5):878-883.

9. Scalea, T. M., Rodriguez, A., Chiu, W. C., Brenneman, F. D., Fallon, W. F., Kato, K. et al. (1999). Focused assessment with sonography for trauma (FAST): results from an international consensus conference. Journal of Trauma and Acute Care Surgery. 1999;46(3): 466-472.

10. Shackford, S. R., Rogers, F. B., Osler, T. M., Trabulsy, M. E., Clauss, D. W., Vane, D. W. Focused abdominal sonogram for trauma: the learning curve of nonradiologist clinicians in detecting hemoperitoneum. Journal of Trauma and Acute Care Surgery, 1999; 46(4):553-564.

11. Branney, S. W., Wolfe, R. E., Moore, E. E., Albert, N. P., Heinig, M., Mestek, M., et al. Quantitative sensitivity of ultrasound in detecting free intraperitoneal fluid. Journal of Trauma and Acute Care Surgery. 1995;39(2):375-380.

12. Willmann, J. K., Roos, J. E., Platz, A., Pfammatter, T., Hilfiker, P. R., Marincek, B., Weishaupt, D. Multidetector CT: detection of active hemorrhage in patients with blunt abdominal trauma. American Journal of Roentgenology, 2002;179(2):437-444.

13. Fabian TC, Croce MA. Abdominal Trauma, Including Indications for Celiotomy. In: Mattox Kl, Feliciano DV, Moore EE, eds. Trauma. New York, NY: McGraw-Hill; 2000. 583-599.

14. Hoff WS, Holevar M, Nagy KK, Patterson L, Young JS, Arrillaga A, Najarian MP, Valenziano CP. Practice Management Guidelines for the Evaluation of Blunt Abdominal Trauma: The EAST Practice Management Guidelines Work Group. Journal of Trauma and Acute Care Surgery. 2002;53:602-615. 